Article

\title{
Cytotoxic Activity of Semi-Synthetic Derivatives of Elatol and Isoobtusol
}

Karen L. Lang ${ }^{1, *}$, Izabella T. Silva ${ }^{1}$, Lara A. Zimmermann ${ }^{1}$, Cíntia Lhullier ${ }^{1}$, Maria V. Mañalich Arana ${ }^{2}$, Jorge A. Palermo ${ }^{2}$, Miriam Falkenberg ${ }^{1}$, Cláudia M. O. Simões ${ }^{1}$, Eloir P. Schenkel ${ }^{1}$ and Fernando J. Durán ${ }^{2}$

1 Department of Pharmaceutical Sciences, Federal University of Santa Catarina, Florianópolis, SC, 88040-970, Brazil; E-Mails: izabellathais@hotmail.com (I.T.S.); lalinhazi@gmail.com (L.A.Z.); clhullier@yahoo.com.br (C.L.); miriam@ccs.ufsc.br (M.F.); claudias@reitoria.ufsc.br (C.M.O.S.); eloirschenkel@gmail.com (E.P.S.)

2 Department of Organic Chemistry, University of Buenos Aires, Buenos Aires, C1428EGA, Argentina; E-Mails: vmanalich@qo.fcen.uba.ar (M.V.M.A.); palermo@qo.fcen.uba.ar (J.A.P.); fduran@qo.fcen.uba.ar (F.J.D.)

* Author to whom correspondence should be addressed; E-Mail: karenluise@gmail.com; Tel.: +55-48-37215076; Fax: +55-48-37219350.

Received: 1 August 2012; in revised form: 12 September 2012 / Accepted: 1 October 2012 / Published: 18 October 2012

\begin{abstract}
In the present study, the in vitro cytotoxic effects of six semi-synthetic derivatives of elatol (1) and isoobtusol (2) were investigated. Chemical modifications were performed on the hydroxyl groups aiming to get derivatives of different polarity, namely the hemisuccinate, carbamate and sulfamate. The structural elucidation of the new derivatives was based on detailed NMR and MS spectroscopic analyses. The in vitro cytotoxicity of compounds $\mathbf{1}$ to $\mathbf{8}$ was evaluated against A459 and RD tumor cell lines with $\mathrm{CC}_{50}$ values ranging from 4.93 to $41.53 \mu \mathrm{M}$. These results suggest that the structural modifications performed on both compounds could be considered a good strategy to obtain more active derivatives.
\end{abstract}

Keywords: elatol; isoobtusol; sesquiterpenes; synthesis; cytotoxic activity 


\section{Introduction}

The oceanic environment has been a vast source of natural products, yielding a wide range of bioactive compounds with diverse mechanisms of action [1]. The genus Laurencia (order Ceremiales, family Rhodomelaceae) is a rich source of halogenated secondary metabolites, predominantly sesquiterpenes, diterpenes, and $\mathrm{C} 15$ non-terpenoids, chemically diverse compounds with great therapeutic potential [2].

Elatol (1) (Figure 1), a halogenated chamigrane sesquiterpene, was isolated for the first time from Laurencia elata by Sims et al. [3]. Several species of Laurencia produce this sesquiterpene as a major secondary metabolite [4-7], especially Laurencia microcladia from which elatol was obtained with the high yield of ca. $10 \%(\mathrm{w} / \mathrm{w})$ from the ethanolic extract of the alga [8]. This compound has displayed antifeedant [9], antifouling [10], antibacterial [6,11], antifungal [12], antiparasitic [13,14] and cytotoxic activity against HeLa and Hep-2 human carcinoma cell lines [15]. A recent investigation [16] showed that elatol caused in vitro an increase in cell numbers at the G1 and the sub-G1 phases, indicating apoptosis induction; and was able to reduce tumor growth in vivo in C57B16 mice inoculated with B16F10 cells.

Isoobtusol (2) (Figure 1) belongs to the same structural class of elatol and was described for the first time from Laurencia obtusa by González et al. [17]. It showed strong antimicrobial activity against several strains, which included human pathogens $[6,12]$.

Figure 1. The structure of Elatol (1) and Isoobtusol (2).

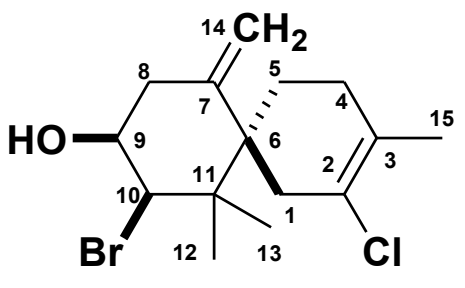

1

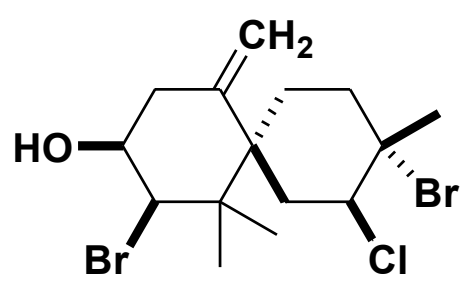

2

In spite of this promising pharmacological profile, the high lipophilicity of elatol and isoobtusol and their consequent low aqueous solubility are limiting aspects for further studies. In order to address these issues, chemical modifications were performed on the hydroxyl groups aiming to get analogues with different polarity. With this objective, six compounds were synthetized by reaction with chlorosulfonyl isocyanate and succinic anhydride to obtain the corresponding carbamates (3 and 6), sulfamates (5 and 8) and hemissuccinates (4 and 7). Such derivatives have been shown to improve not only the water solubility, but in some cases also the cytotoxic activity of the compounds, as its amphiphilic character is supposed to enhance absorption in biological systems $[18,19]$. In this way, these compounds were evaluated for in vitro cytotoxic activity against lung (A549) and embryo rhabdomyosarcome (RD) tumor cells, and a preliminary structure-activity relationship (SAR) is presented. 


\section{Results and Discussion}

\subsection{Chemistry}

Six derivatives of elatol (1) and isoobtusol (2) were synthesized by the routes described in Schemes 1 and 2, and their cytotoxic activity was evaluated.

Scheme 1. Synthesis of elatol derivatives (3), (4) and (5).
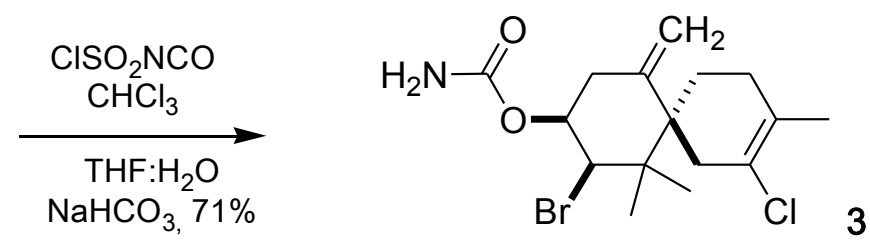<smiles>C=C1CC(O)C(Br)C(C)(C)[C@]12CCC(C)=C(Cl)C2</smiles>

1<smiles>C=C1CC(OC(=O)CCC(=O)O)C(Br)C(C)(C)[C@]12CCC(C)=C(Cl)C2</smiles>
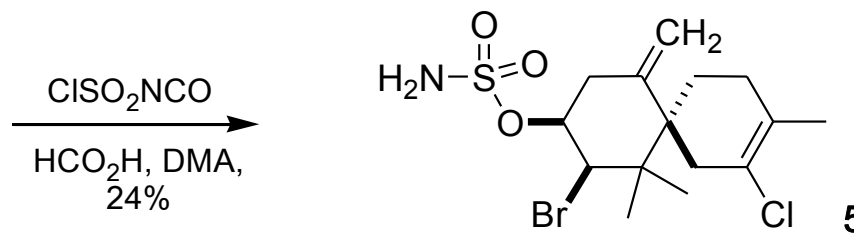

Scheme 2. Synthesis of isoobtusol derivatives (6), (7) and (8).

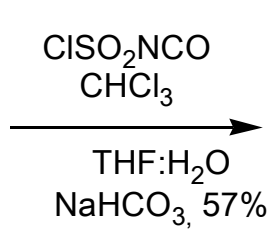<smiles>C=C1CC(OC(N)=O)C(Br)C(C)(C)C12CCC(C)(Br)C(Cl)C2</smiles><smiles>C=C1CC(O)C(Br)C(C)(C)C12CCC(C)(Br)C(Cl)C2</smiles>

2<smiles>C=C1CC(OC(=O)CCC(=O)O)C(Br)C(C)(C)C12CC[C@@](C)(Br)C(Cl)C2</smiles><smiles>C=C1CC(OS(N)(=O)=O)C(Br)C(C)(C)C12CCC(C)(Br)C(Cl)C2</smiles>

The carbamate derivative (3) was prepared by treatment of (1) with chlorosulfonyl isocyanate as described previously by Bandyopadhyay et al. [20]. Compound (4) was prepared by the reaction of (1) 
with succinic anhydride in presence of DMAP, pyridine and $\mathrm{CH}_{2} \mathrm{Cl}_{2}$. Compound (5) was obtained by reaction with chlorosulfonyl isocyanate in the presence of formic acid and DMA [21]. Compounds (6), (7) and (8) were obtained from (2) according to the same methods used for the preparation of compounds (3), (4) and (5), respectively. The compounds were purified by silica gel column chromatography with hexane/ethyl acetate as eluant and their structures confirmed by IR, ${ }^{1} \mathrm{H}-\mathrm{NMR}$, ${ }^{13} \mathrm{C}-\mathrm{NMR}, 2 \mathrm{D}-\mathrm{NMR}$ and MS.

\subsection{Cytotoxic Activity}

To quantify the cytotoxic effects of elatol (1), isoobtusol (2) and their synthetic derivatives (3) to (8), the $\mathrm{CC}_{50}$ value of each compound was measured $\left(\mathrm{CC}_{50}\right.$ is defined as the concentration that reduced cell growth by $50 \%$ after $48 \mathrm{~h}$ ). Table 1 shows the $\mathrm{CC}_{50}$ values obtained for the tested compounds against human non-small cell lung tumor (A549) and human embryo rhabdomyosarcome (RD) cells.

Table 1. Cytotoxicity of compounds $\mathbf{1}$ to $\mathbf{8}$ against two different human tumor cell lines.

\begin{tabular}{ccc}
\hline \multirow{2}{*}{ Compound } & \multicolumn{2}{c}{ Cell lines $\left[\mathbf{C C}_{\mathbf{5 0}}{ }^{\mathbf{a}} \boldsymbol{( \boldsymbol { \mu M } ) ]}\right.$} \\
\cline { 2 - 3 } $\mathbf{1}$ & $\mathbf{A 5 4 9}$ & $\mathbf{R D}$ \\
$\mathbf{2}$ & $14.56 \pm 0.19$ & $11.22 \pm 1.63$ \\
$\mathbf{3}$ & $21.93 \pm 1.27$ & $6.24 \pm 1.11$ \\
$\mathbf{4}$ & $39.05 \pm 4.81$ & $13.26 \pm 0.76$ \\
$\mathbf{5}$ & $>100$ & $21.23 \pm 7.16$ \\
$\mathbf{6}$ & $39.57 \pm 2.07$ & $41.53 \pm 0.36$ \\
$\mathbf{7}$ & $10.74 \pm 2.52$ & $23.83 \pm 5.28$ \\
$\mathbf{8}$ & $23.85 \pm 5.21$ & $4.93 \pm 0.52$ \\
Paclitaxel & $0.260 \pm 0.027$ & $20.48 \pm 1.44$ \\
\end{tabular}

Values represent the mean \pm standard deviations of three independent experiments; ${ }^{\text {a }}$ Cytotoxicity was determined by MTT assay on each human tested cancer cell line.

Elatol (1) and isoobtusol (2) exhibited significant cytotoxicity against the tested human tumor cell lines with $\mathrm{CC}_{50}$ values ranging from 6.24 to $14.24 \mu \mathrm{M}$. Elatol was approximately twice more active against A549 cells, while, on the other hand, isoobtusol was approximately twice more active against $\mathrm{RD}$ cells. In relation to the elatol derivatives (4) and (5) and the isoobtusol derivatives (6) and (8), the modifications on the hydroxyl group at C-9 lead to a reduction in the observed cytotoxicity. Carbamate derivatives (3) and (6) were approximately three times less active than the original compounds against A549 cells, while derivative (3) was just slightly less active than elatol itself towards RD cells. An increase of the cytotoxic activity was observed for compound (7), which is the hemisuccinate of isoobtusol, since it was more cytotoxic than its precursor against $\mathrm{A} 549$ cells $\left(\mathrm{CC}_{50}=10.74 \mu \mathrm{M}\right)$ and also against $\mathrm{RD}$ cells $\left(\mathrm{CC}_{50}=4.93 \mu \mathrm{M}\right)$. It is interesting to note that the hemissuccinate derivative of isoobtusol (7) and isoobutusol itself (2) showed similar profiles being more active against RD than to A549 cells. Nevertheless, the hemisuccinate derivative of elatol (4) as well the sulfamate derivatives (5) and (8) were less active than the original compounds to both cell lines. These findings suggest that the substituent at C-9 plays an important role for the cytotoxic activity against these cell lines, and the best results were observed with the free hydroxyl or the hemisuccinate group. 


\section{Experimental}

\subsection{General}

NMR spectra were recorded in $\mathrm{CDCl}_{3}$ or $\mathrm{CD}_{3} \mathrm{OD}$ at 500.13 and $125.13 \mathrm{MHz}$ for ${ }^{1} \mathrm{H}$ and ${ }^{13} \mathrm{C}$, respectively, on a Bruker Avance $2500 \mathrm{MHz}$ NMR spectrometer with TMS or the signal of residual non-deuterated solvent as internal standard. High-resolution ESI (ESI-HR-MS) mass spectra were recorded on a Bruker-Daltronics MicroTOF-Q II mass spectrometer. IR spectra were obtained on a Shimadzu Prestige 2 instrument by using $\mathrm{KBr}$ pellets. Column chromatography was performed using

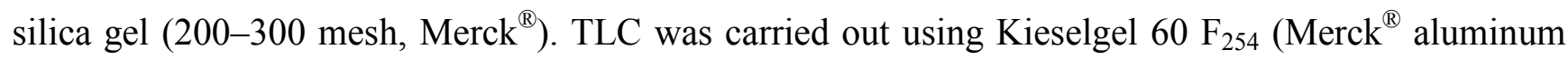
support plates). All solvents were of analytical grade and were purchased from Nuclear ${ }^{\circledR}$. Chemical reagents, as well as deuterated solvents for NMR experiments, were purchased from Aldrich Chemical Company (Milwaukee, Wisconsin, USA).

\subsection{Algal Material}

Laurencia microcladia was collected by hand in March 2010 at the lower intertidal zone of Praia da Sepultura $\left(27^{\circ} 07^{\prime} 54^{\prime \prime} \mathrm{S}\right.$ and $\left.48^{\circ} 31^{\prime} 40^{\prime \prime} \mathrm{W}\right)$, Santa Catarina, Southern Brazilian coast. Voucher samples are kept at the Herbarium of the Department of Botany, Federal University of Santa Catarina (FLOR 14516-14520).

Fresh material of L. microcladia $(1 \mathrm{~kg})$ was exhaustively extracted with ethanol at room temperature for 3 days (three times). The concentrated extract $(5 \mathrm{~g})$ was partitioned with ethyl acetate and water, affording $2 \mathrm{~g}$ of aqueous fraction and $3 \mathrm{~g}$ of ethyl acetate fraction. This latter was fractionated as previously described by Lhullhier et al. [8] to yield pure compounds $\mathbf{1}$ (500 mg) and 2 (400 mg).

\subsection{Synthesis}

\subsubsection{Elatol 9-Carbamate (3)}

To a solution of $1(100 \mathrm{mg}, 0.3 \mathrm{mmol})$ in $\mathrm{CHCl}_{3}(0.4 \mathrm{~mL})$ chlorosulfonyl isocyanate was added dropwise $(0.08 \mathrm{~mL}, 0.9 \mathrm{mmol})$ at $20{ }^{\circ} \mathrm{C}$ under nitrogen atmosphere with vigorous stirring. The reaction mixture was left overnight during $16 \mathrm{~h}$, then, a solution of $\mathrm{THF} / \mathrm{H}_{2} \mathrm{O}(1 / 1 \mathrm{v} / \mathrm{v}, 2 \mathrm{~mL})$ was added, followed by a saturated solution of $\mathrm{NaHCO}_{3}(1 \mathrm{~mL})$. The obtained suspension was stirred for an additional $2 \mathrm{~h}$ at $20^{\circ} \mathrm{C}$ and then diluted with chloroform $(10 \mathrm{~mL})$. The organic phase was separated and washed with $\mathrm{H}_{2} \mathrm{O}(2 \times 10 \mathrm{~mL})$, dried over anhydrous sodium sulphate and evaporated under reduced pressure. The resulting crude product was purified by column chromatography on silica gel using hexane/EtOAc (8:2) and afforded $80 \mathrm{mg}$ (71\%) of carbamate 3 as a white solid. Mp: $79-81{ }^{\circ} \mathrm{C}$; IR (KBr): 3470, 3240, 1705, 1600, 1385, 1334, 1078, $1055 \mathrm{~cm}^{-1},{ }^{1} \mathrm{H}$ NMR (500 $\left.\mathrm{MHz}, \mathrm{CDCl}_{3}\right)$ :

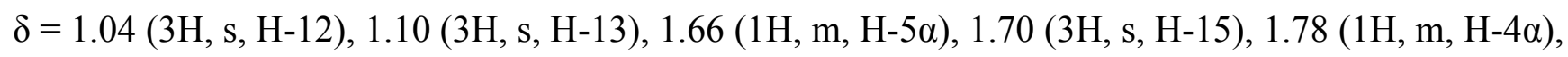
$1.81(1 \mathrm{H}, \mathrm{m}, \mathrm{H}-5 \beta), 1.97(1 \mathrm{H}, \mathrm{m}, \mathrm{H}-4 \beta), 2.36(1 \mathrm{H}, \mathrm{d}, J=17.3 \mathrm{~Hz}, \mathrm{H}-1 \beta), 2.51(1 \mathrm{H}, \mathrm{dd}, J=15.0 / 2.8 \mathrm{~Hz}$, $\mathrm{H}-8 \alpha), 2.60(1 \mathrm{H}, \mathrm{d}, J=15.0 \mathrm{~Hz}, \mathrm{H}-8 \beta), 2.61(1 \mathrm{H}, \mathrm{d}, J=17.3 \mathrm{~Hz}, \mathrm{H}-1 \alpha), 4.53(1 \mathrm{H}, \mathrm{d}, J=3.4 \mathrm{~Hz}$, $\mathrm{H}-10), 4.77$ (1H, s, H-14ß), $5.04(1 \mathrm{H}, \mathrm{s}, \mathrm{H}-14 \alpha), 5.15(1 \mathrm{H}, \mathrm{dd}, J=6.3 / 3.4 \mathrm{~Hz}, \mathrm{H}-9) .{ }^{13} \mathrm{C}$ NMR $\left(126 \mathrm{MHz}, \mathrm{CDCl}_{3}\right): \delta=19.6(\mathrm{C}-15), 20.4$ (C-12), 24.4 (C-13), 25.7 (C-5), 29.4 (C-4), 37.1 (C-8), 38.8 (C-1), 43.6 (C-11), 49.2 (C-6), 63.4 (C-10), 74.5 (C-9), 116.0 (C-14), 124.2 (C-2), 128.3 (C-3), 140.8 
(C-7), $156.0\left(\mathrm{C}-1^{\prime}\right)$; ESI-MS $m / z$ 398.0496 [M + Na $]^{+}$(calcd for $\mathrm{C}_{16} \mathrm{H}_{23} \mathrm{BrClNNaO}_{2}, 398.0493$ ), 400.0482 [M $+2+\mathrm{Na}]^{+}$(calcd. 400.0471), $402.0444[\mathrm{M}+4+\mathrm{Na}]^{+}$(calcd. 402.0449), observed isotopic pattern (398/400/402): 79/100/22, (theoretical: 76/100/25).

\subsubsection{Elatol 9-Hemisuccinate (4)}

To a solution of $1(50 \mathrm{mg}, 0.15 \mathrm{mmol})$ in $\mathrm{CH}_{2} \mathrm{Cl}_{2}(1 \mathrm{~mL})$ and pyridine $(0.1 \mathrm{~mL})$, succinic anhydride $(150 \mathrm{mg}, 1.5 \mathrm{mmol})$ and a catalytic amount of DMAP were added with stirring at $20^{\circ} \mathrm{C}$. After $24 \mathrm{~h}$, the mixture was diluted with $\mathrm{CH}_{2} \mathrm{Cl}_{2}(10 \mathrm{~mL})$ and washed with $\mathrm{HCl} 1 \mathrm{~N}(2 \times 10 \mathrm{~mL})$. The organic phase was dried with anhydrous sodium sulfate and evaporated under reduced pressure. The resulting crude product was purified by flash column chromatography on silica gel using hexane/EtOAc (8:2), to give the hemisuccinate 4 (35 mg, 69.8\% yield) as yellow oil. IR (KBr): $3100,1730,1160 \mathrm{~cm}^{-1}$; ${ }^{1} \mathrm{H}$ NMR (500 MHz, $\left.\mathrm{CDCl}_{3}\right): \delta=1.06(3 \mathrm{H}, \mathrm{s}, \mathrm{H}-12), 1.10(3 \mathrm{H}, \mathrm{s}, \mathrm{H}-13), 1.65(1 \mathrm{H}, \mathrm{m}, \mathrm{H}-5 \alpha), 1.70(3 \mathrm{H}$, s, H-15), $1.80(1 \mathrm{H}, \mathrm{m}, \mathrm{H}-4 \alpha), 1.81(1 \mathrm{H}, \mathrm{m}, \mathrm{H}-5 \beta), 1.97(1 \mathrm{H}, \mathrm{m}, \mathrm{H}-4 \beta), 2.36(1 \mathrm{H}, \mathrm{d}, J=17.3 \mathrm{~Hz}$, $\mathrm{H}-1 \beta), 2.43(1 \mathrm{H}, \mathrm{dd}, J=15.0 / 2.7 \mathrm{~Hz}, \mathrm{H}-8 \alpha), 2.61(1 \mathrm{H}, \mathrm{d}, J=15.0 \mathrm{~Hz}, \mathrm{H}-8 \beta), 2.62(1 \mathrm{H}, \mathrm{d}, J=17.3 \mathrm{~Hz}$,

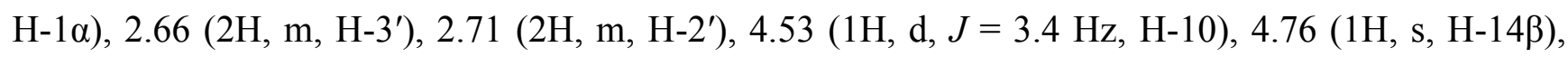

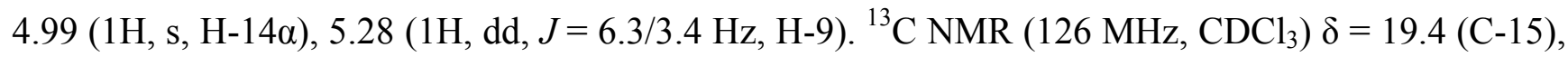
20.2 (C-12), 24.2 (C-13), 25.6 (C-5), 28.8 (C-2'), 29.1 (C-3'), 29.4 (C-4), 36.6 (C-8), 38.6 (C-1), 43.3 (C-11), 48.9 (C-6), 62.8 (C-10), 74.1 (C-9), 116.0 (C-14), 124.0 (C-2), 128.1 (C-3), 140.3 (C-7), $171.1\left(\mathrm{C}-1^{\prime}\right), 177.4\left(\mathrm{C}-4^{\prime}\right)$; ESI-MS $m / z 455.0591[\mathrm{M}+\mathrm{Na}]^{+}$(calcd for $\mathrm{C}_{19} \mathrm{H}_{26} \mathrm{BrClNaO}_{4}, 455.0595$ ), 457.0580 [M $+2+\mathrm{Na}^{+}$(calcd. 457.0574), $459.0557[\mathrm{M}+4+\mathrm{Na}]^{+}$(calcd. 459.0555), observed isotopic pattern (455/457/459): 73/100/24, (theoretical: 76/100/26).

\subsubsection{Elatol 9-Sulfamate (5)}

Formic acid $(0.03 \mathrm{~mL}, 0.9 \mathrm{mmol})$ was added dropwise to chlorosulfonyl isocyanate $(0.08 \mathrm{~mL}$, $0.9 \mathrm{mmol})$ at $0{ }^{\circ} \mathrm{C}$ with rapid stirring. Gas evolution was observed during the addition process. The resulting viscous suspension was stirred for $18 \mathrm{~h}$ at room temperature. The reaction mixture was cooled to $0{ }^{\circ} \mathrm{C}$, DMA $(0.2 \mathrm{~mL})$ was added, and the solution was stirred for $5 \mathrm{~min}$. A solution of $\mathbf{1}$ $(0.3 \mathrm{mmol}, 100 \mathrm{mg})$ in DMA $(0.5 \mathrm{~mL})$ was added dropwise, and the reaction was allowed to warm to $20{ }^{\circ} \mathrm{C}$ over a $1 \mathrm{~h}$ period. The reaction was quenched by the successive addition of EtOAc $(10 \mathrm{~mL})$ and brine $(5 \mathrm{~mL})$. The mixture was poured on EtOAc $(20 \mathrm{~mL})$ and water $(10 \mathrm{~mL})$, the organic phase was collected, and the aqueous layer was extracted with EtOAc $(20 \mathrm{~mL})$. The combined organic extracts were washed with brine $(2 \times 10 \mathrm{~mL})$, dried over $\mathrm{Na}_{2} \mathrm{SO}_{4}$ and concentrated under reduced pressure. Purification of the residue by flash column chromatography on silica gel using hexane/EtOAc (7:3) gave the sulfamidate 5 (30.5 mg, 24\% yield) as a white solid. Mp: 199-202 ${ }^{\circ} \mathrm{C}$; IR (KBr): 3333, 3265, 1557, 1350, 1196, 1140, $900 \mathrm{~cm}^{-1} ;{ }^{1} \mathrm{H}$ NMR (500 MHz, $\left.\mathrm{CDCl}_{3}\right): \delta=1.02(3 \mathrm{H}, \mathrm{s}, \mathrm{H}-12), 1.10(3 \mathrm{H}, \mathrm{s}$,

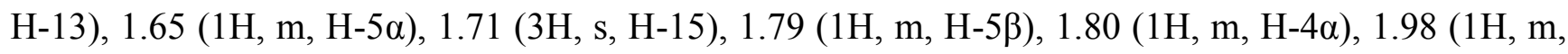

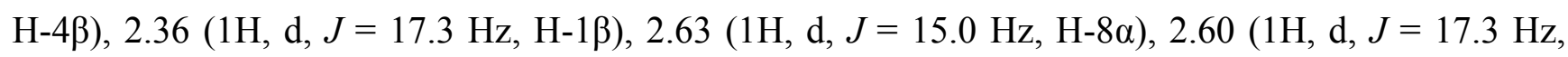

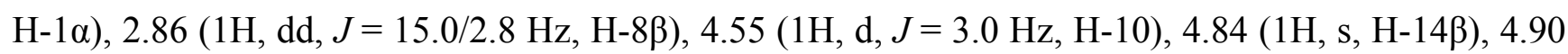
$(1 \mathrm{H}, \mathrm{dd}, J=6.0 / 3.0 \mathrm{~Hz}, \mathrm{H}-9), 5.17(1 \mathrm{H}, \mathrm{s}, \mathrm{H}-14 \alpha) ;{ }^{13} \mathrm{C} \mathrm{NMR}\left(126 \mathrm{MHz}, \mathrm{CDCl}_{3}\right): \delta=19.4(\mathrm{C}-15), 20.1$ (C-12), 24.3 (C-13), 25.6 (C-5), 29.4 (C-4), 37.1 (C-8), 38.6 (C-1), 43.6 (C-11), 49.4 (C-6), 62.6 (C-10), 82.2 (C-9), 117.1 (C-14), 124.0 (C-2), 128.2 (C-3), 139.2 (C-7); ESI-MS m/z 429.0587 
$\left[\mathrm{M}+\mathrm{NH}_{4}\right]^{+}$(calcd for $\mathrm{C}_{15} \mathrm{H}_{27} \mathrm{BrClN}_{2} \mathrm{O}_{3} \mathrm{~S}, 429.0608$ ), $431.0575\left[\mathrm{M}+2+\mathrm{NH}_{4}\right]^{+}$(calcd. 431.0587), 433.0565 [M $\left.+4+\mathrm{NH}_{4}\right]^{+}$(calcd. 433.0563), observed isotopic pattern (429/431/433): 73/100/28, (theoretical: $73 / 100 / 29$ ).

\subsubsection{Isoobtusol 9-Carbamate (6)}

Compound 6 was similarly prepared, according to the procedure of $\mathbf{3}$. To a solution of $\mathbf{2}$ (100 $\mathrm{mg}$, $0.24 \mathrm{mmol})$ in $\mathrm{CHCl}_{3}(0.4 \mathrm{~mL})$ chlorosulfonyl isocyanate $(0.06 \mathrm{~mL}, 0.74 \mathrm{mmol})$ was added dropwise at $20{ }^{\circ} \mathrm{C}$ under nitrogen atmosphere with vigorous stirring. The reaction mixture was left overnight during $16 \mathrm{~h}$, and then a solution of $\mathrm{THF} / \mathrm{H}_{2} \mathrm{O}(1 / 1 \mathrm{v} / \mathrm{v}, 1.5 \mathrm{~mL})$ was added, followed by a saturated solution of $\mathrm{NaHCO}_{3}(0.7 \mathrm{~mL})$. The obtained suspension was stirred for an additional $2 \mathrm{~h}$ at $20{ }^{\circ} \mathrm{C}$ and then diluted with chloroform $(10 \mathrm{~mL})$. The organic phase was separated and washed with $\mathrm{H}_{2} \mathrm{O}$ $(2 \times 10 \mathrm{~mL})$, dried over anhydrous sodium sulpfate and evaporated under reduced pressure. The resulting crude product was purified by column chromatography on silica gel using hexane/EtOAc (8:2) afforded $62.4 \mathrm{mg}(57 \%)$ of carbamate 6 as white solid. Mp: $158-160{ }^{\circ} \mathrm{C}$; IR (KBr): 3472,3241 , 1728, 1604, 1324, 1328, $1064 \mathrm{~cm}^{-1} ;{ }^{1} \mathrm{H}$ NMR (500 MHz, $\left.\mathrm{CDCl}_{3}\right): \delta=1.11(3 \mathrm{H}, \mathrm{s}, \mathrm{H}-13), 1.32(3 \mathrm{H}, \mathrm{s}$, H-12), 1.80 (1H, ddd, $J=14.0 / 6.5 / 3.5 \mathrm{~Hz}, \mathrm{H}-4 \alpha), 1.85(1 \mathrm{H}, \mathrm{m}, \mathrm{H}-5 \alpha), 1.92(3 \mathrm{H}, \mathrm{s}, \mathrm{H}-15), 2.06(1 \mathrm{H}, \mathrm{td}$, $J=14.0 / 3.5 \mathrm{~Hz}, \mathrm{H}-4 \beta), 2.24(1 \mathrm{H}, \mathrm{m}, \mathrm{H}-5 \beta), 2.41(1 \mathrm{H}, \mathrm{dd}, J=12.2 / 3.4 \mathrm{~Hz}, \mathrm{H}-8 \alpha), 2.82(1 \mathrm{H}, \mathrm{dd}$, $J=15 / 3.7 \mathrm{~Hz}, \mathrm{H}-1 \beta), 2.92(1 \mathrm{H}, \mathrm{t}, J=12.2 \mathrm{~Hz}, \mathrm{H}-8 \beta), 3.17(1 \mathrm{H}, \mathrm{d}, J=15 \mathrm{~Hz}, \mathrm{H}-1 \alpha), 4.45$ (1H, m, H-2), 4.46 (1H, dd, $J=3.4 / 1.9$ Hz, H-10), 4.73 (1H, m, H-9), 4.98 (1H, s, H-14), 5.21 (1H, s, H-14); ${ }^{13} \mathrm{C}$ NMR (126 MHz, $\left.\mathrm{CDCl}_{3}\right): \delta=24.6$ (C-12), 25.1 (C-13), 25.6 (C-4), 33.1 (C-15), 33.3 (C-5), 34.0 (C-1), 35.4 (C-8), 43.7 (C-6), 44.1 (C-11), 65.2 (C-2), 67.3 (C-10), 71.1 (C-3), 72.0 (C-9), 114.6 (C-14), 146.9 (C-7), 155.6 (C-1'); ESI-MS m/z 477.9773 [M + $\mathrm{Na}^{+}$(calcd for $\left.\mathrm{C}_{16} \mathrm{H}_{24} \mathrm{Br}_{2} \mathrm{ClNNaO}_{2}, 477.9754\right), 479.9736[\mathrm{M}+2+\mathrm{Na}]^{+}$(calcd. 479.9733), $481.9727[\mathrm{M}+4+\mathrm{Na}]^{+}$ (calcd. 481.9712), $483.9684[\mathrm{M}+6+\mathrm{Na}]^{+}$(calcd. 483.9692), observed isotopic pattern (477/479/481/483): 43/100/69/13, (theoretical: 44/100/70/14).

\subsubsection{Isoobtusol 9-Hemisuccinate (7)}

Compound 7 was prepared in a similar way as 4 . To a solution of $2(50 \mathrm{mg}, 0.12 \mathrm{mmol})$ in $\mathrm{CH}_{2} \mathrm{Cl}_{2}$ $(1 \mathrm{~mL})$ and pyridine $(0.1 \mathrm{~mL})$, succinic anhydride $(121 \mathrm{mg}, 1.2 \mathrm{mmol})$ and a catalytic amount of DMAP were added with stirring at $20{ }^{\circ} \mathrm{C}$. After $24 \mathrm{~h}$, the mixture was diluted with $\mathrm{CH}_{2} \mathrm{Cl}_{2}(10 \mathrm{~mL})$ and washed with $\mathrm{HCl} 1 \mathrm{~N}(2 \times 10 \mathrm{~mL})$. The organic phase was dried with anhydrous sodium sulfate and evaporated under reduced pressure. The resulting crude product was purified by flash column chromatography on silica gel using hexane/EtOAc (8:2), to give the hemisuccinate 7 (31 $\mathrm{mg}, 62 \%$ yield) as with solid. Mp: $116-118{ }^{\circ} \mathrm{C}$; IR (KBr): $3100,1738,1184 \mathrm{~cm}^{-1} ;{ }^{1} \mathrm{H}$ NMR (500 MHz CDCl 3 :MeOD, 2:1): $\delta=5.21(1 \mathrm{H}, \mathrm{s}, \mathrm{H}-14 \alpha), 5.0(1 \mathrm{H}, \mathrm{s}, \mathrm{H}-14 \beta), 4.85$ (1H, m, H-9), 4.46 (1H, br s, H-2), 4.42 (1H, dd, $J=3.4 / 1.9 \mathrm{~Hz}, \mathrm{H}-10), 3.16(1 \mathrm{H}, \mathrm{d}, J=15 \mathrm{~Hz}, \mathrm{H}-1 \alpha), 2.95(1 \mathrm{H}, \mathrm{t}, J=12.2 \mathrm{~Hz}, \mathrm{H}-8 \beta), 2.81(1 \mathrm{H}, \mathrm{dd}$, $J=15 / 3.7 \mathrm{~Hz}, \mathrm{H}-1 \beta), 2.67$ (2H, overlapped, H-3'), 2.62 (2H, overlapped, H-2'), 2.41 (1H, m, H-8 $\alpha$ ),

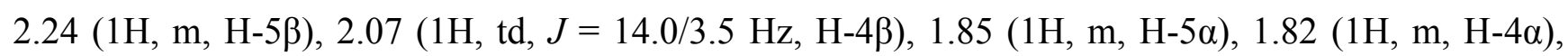
$1.31(3 \mathrm{H}, \mathrm{s}, \mathrm{H}-12), 1.11(3 \mathrm{H}, \mathrm{s}, \mathrm{H}-13)$; ${ }^{13} \mathrm{C} \mathrm{NMR}\left(126 \mathrm{MHz}, \mathrm{CDCl}_{3}: \mathrm{MeOD}, 2: 1\right): \delta=174.7\left(\mathrm{C}-4^{\prime}\right)$, 172.0 (C-1'), 146.8 (C-7), 114.9 (C-14), 72.0 (C-9), 71.2 (C-3), 66.3 (C-10), 65.3 (C-2), 43.9 (C-6), 35.2 (C-8), 34.1 (C-1), 33.4 (C-5), 33.1 (C-15), 29.0 (C-3'), 28.9 (C-2'), 25.7 (C-4), 25.1 (C-13), 
24.6 (C-12); ESI-MS m/z 530.0295 [M + $\left.\mathrm{NH}_{4}\right]^{+}$(calcd for $\mathrm{C}_{19} \mathrm{H}_{31} \mathrm{Br}_{2} \mathrm{ClNO}_{4}, 530.0303$ ), 532.0276 $\left[\mathrm{M}+2+\mathrm{NH}_{4}\right]^{+}$(calcd. 532.0282), 534.0260 $\left[\mathrm{M}+4+\mathrm{NH}_{4}\right]^{+}$(calcd. 534.0262), 536.0175 $\left[\mathrm{M}+6+\mathrm{NH}_{4}\right]^{+}$(calcd. 536.0244), observed isotopic pattern (530/532/534/536): 42/100/74/11, (theoretical: 43/100/72/15).

\subsubsection{Isoobtusol 9-Sulfamate (8)}

Compound 8 was prepared according to the procedure of $\mathbf{5}$. Formic acid $(0.03 \mathrm{~mL}, 0.73 \mathrm{mmol})$ was added dropwise to chlorosulfonyl isocyanate $(0.06 \mathrm{~mL}, 0.73 \mathrm{mmol})$ at $0{ }^{\circ} \mathrm{C}$ with rapid stirring. Gas evolution was observed during the addition process. The resulting viscous suspension was stirred for $18 \mathrm{~h}$ at room temperature. The reaction mixture was cooled to $0{ }^{\circ} \mathrm{C}$, DMA $(0.2 \mathrm{~mL})$ was added, and the solution was stirred for $5 \mathrm{~min}$. A solution of $2(100 \mathrm{mg}, 0.24 \mathrm{mmol})$ in DMA $(0.3 \mathrm{~mL})$ was added dropwise, and the reaction was allowed to warm to $20{ }^{\circ} \mathrm{C}$ over a $1 \mathrm{~h}$ period. The reaction was quenched by the successive addition of EtOAc $(10 \mathrm{~mL})$ and brine $(5 \mathrm{~mL})$. The mixture was poured on EtOAc $(20 \mathrm{~mL})$ and water $(10 \mathrm{~mL})$, the organic phase was collected, and the aqueous layer was extracted with EtOAc $(20 \mathrm{~mL})$. The combined organic extracts were washed with brine $(2 \times 10 \mathrm{~mL})$, dried over $\mathrm{Na}_{2} \mathrm{SO}_{4}$ and concentrated under reduced pressure. Purification of the residue by flash column chromatography on silica gel using hexanes/EtOAc (7:3) gave the sulfamidate 8 (15.0 mg, $13 \%$ yield) as a white solid. Mp: $143-145^{\circ} \mathrm{C}$; IR (KBr): 3410, 3282, 1520, 1366, 1182, 1167, $933 \mathrm{~cm}^{-1}$; ${ }^{1} \mathrm{H}$ NMR $\left(500 \mathrm{MHz}, \mathrm{CDCl}_{3}\right): \delta=5.26(1 \mathrm{H}, \mathrm{s}, \mathrm{H}-14 \alpha), 5.03(1 \mathrm{H}, \mathrm{s}, \mathrm{H}-14 \beta), 4.60(1 \mathrm{H}, \mathrm{m}, \mathrm{H}-9)$, $4.45(1 \mathrm{H}, \mathrm{m}, \mathrm{H}-2), 4.44(1 \mathrm{H}, \mathrm{dd}, J=4.0 / 2.0 \mathrm{~Hz}, \mathrm{H}-10), 3.16(1 \mathrm{H}, \mathrm{d}, J=15 \mathrm{~Hz}, \mathrm{H}-1 \alpha), 3.04(1 \mathrm{H}, \mathrm{t}$, $J=12.5 \mathrm{~Hz}, \mathrm{H}-8 \beta), 2.82(1 \mathrm{H}, \mathrm{dd}, J=15.6 / 3.2 \mathrm{~Hz}, \mathrm{H}-1 \beta), 2.58(1 \mathrm{H}, \mathrm{dd}, J=12.5 / 2.0 \mathrm{~Hz}, \mathrm{H}-8 \alpha), 2.23$ (1H, m, H-5ß), 2.07 (1H, dt, $J=14.0 / 2.0 \mathrm{~Hz}, \mathrm{H}-4 \beta), 1.86(1 \mathrm{H}, \mathrm{m}, \mathrm{H}-5 \alpha), 1.80(1 \mathrm{H}, \mathrm{m}, \mathrm{H}-4 \alpha), 1.34$ $(3 \mathrm{H}, \mathrm{s}, \mathrm{H}-12), 1.10(3 \mathrm{H}, \mathrm{s}, \mathrm{H}-13) ;{ }^{13} \mathrm{C} \mathrm{NMR}\left(126 \mathrm{MHz}, \mathrm{CDCl}_{3}\right): \delta=146.5$ (C-7), $115.5(\mathrm{C}-14)$, 77.9 (C-9), 70.9 (C-3), 66.3 (C-10), 65.2 (C-2), 44.0 (C-6), 36.2 (C-8), 34.1 (C-1), 33.3 (C-5),

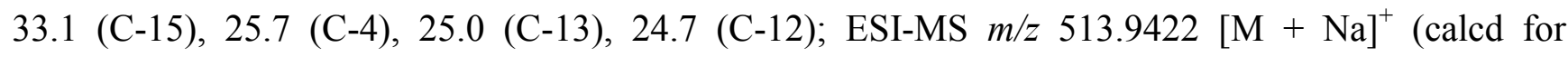
$\left.\mathrm{C}_{15} \mathrm{H}_{24} \mathrm{Br}_{2} \mathrm{ClNNaO}_{3} \mathrm{~S}, 513.9424\right), 515.9419[\mathrm{M}+2+\mathrm{Na}]^{+}$(calcd. 515.9403), $517.9378[\mathrm{M}+4+\mathrm{Na}]^{+}$ (calcd. 517.9381), 519.9355 $[\mathrm{M}+6+\mathrm{Na}]^{+}$(calcd. 519.9358), observed isotopic pattern (513/515/517/519): 73/100/28/2, (theoretical: 73/100/29/2).

\subsection{Cell Lines}

The human embryo rhabdomyosarcome cells (RD) were obtained from Adolfo Lutz Institute, São Paulo, SP, Brazil. The human non-small cell lung cancer (A549 cells) were kindly provided by Dr. Rosina Gironès from the Microbiology Department of the University of Barcelona, Spain.

\subsection{MTT Assay}

RD and A549 cells were grown in minimal essential medium (MEM, Cultilab, São Paulo, Brazil). Both cell lines were supplemented with $10 \%$ fetal bovine serum, and $100 \mathrm{U} / \mathrm{mL}$ penicillin $\mathrm{G}, 100 \mu \mathrm{g} / \mathrm{mL}$ streptomycin, and $25 \mu \mathrm{g} / \mathrm{mL}$ amphotericin B (Gibco, São Paulo, Brazil). Cell cultures were kept in tissue culture flasks in a humidified atmosphere of $5 \% \mathrm{CO}_{2}$ at $37{ }^{\circ} \mathrm{C}$. The effect of the samples treatment on proliferation of RD and A549 cells was measured by the MTT [3-(4,5-dimethylthiazol-2-yl)-2, 
5-diphenyl tetrazolium bromide] assay [22]. Approximately 104 cells were plated per well in 96-well plates and treated with different concentrations of each sample. After $48 \mathrm{~h}$ at $37^{\circ} \mathrm{C}$, the medium was removed, $50 \mu \mathrm{L}$ of MTT reagent $(1 \mathrm{mg} / \mathrm{mL})$ were added to each well, and cells were further incubated at $37^{\circ} \mathrm{C}$ for more $4 \mathrm{~h}$. The MTT solution was removed, $100 \mu \mathrm{L}$ of dimethyl sulfoxide (Nuclear, Brazil) were added to each well to dissolve formazan crystals, and the plates were gently shaken, whereby crystals were completely dissolved. The absorbances were read on a multiwell spectrophotometer (Tecan, Grödig, Austria) at $540 \mathrm{~nm}$. The 50\% cytotoxic concentration (CC 50) of each sample was defined as the concentration that reduced cell viability by $50 \%$ when compared to untreated controls. Paclitaxel ( 0 to $10 \mu \mathrm{M}$, Glenmark, Brazil) was used as positive control (purity $>98 \%$ ).

\subsection{Statistical Analysis}

The mean \pm standard deviations are representative of three independent experiments. For determination of CC 50 values non-linear regressions of concentration-response curves were used.

\section{Conclusions}

The preparation and characterization of new derivatives of elatol and isoobtusol was described as well as their in vitro inhibitory effects on two human tumor cell lines growth. The obtained results will stimulate the introduction of further structural modifications in these natural products in order to enhance these effects.

\section{Acknowledgements}

The authors gratefully acknowledge the financial support of this study and also the research stipends from the Conselho Nacional de Desenvolvimento Científico e Tecnológico (CNPq, MCT, Brazil) to L.A.Z., I.T.S., C.M.O.S and E.P.S, from the Coordenação de Aperfeiçoamento de Pessoal de Nível Superior (CAPES, MEC, Brazil) to K.L.L., and from the Consejo Nacional de Investigaciones Científicas y Técnicas (CONICET, Argentina) and UBA (Universidad de Buenos Aires, Argentina) to M.V.M.A, J.A.P. and F.J.D. We are also grateful to Gabriela M. Cabrera for the ESI-MS analysis, and to Paulo A. Horta (Department of Botany, Federal University of Santa Catarina) for the identification of the alga.

\section{References}

1. Mayer, A.M.; Rodríguez, A.D.; Berlinck, R.G.; Hamann, M.T. Marine pharmacology in 2005-6: Marine compounds with anthelmintic, antibacterial, anticoagulant, antifungal, anti-inflammatory, antimalarial, antiprotozoal, antituberculosis, and antiviral activities; affecting the cardiovascular, immune and nervous systems, and other miscellaneous mechanisms of action. Biochim. Biophys. Acta 2009, 1790, 283-308.

2. Blunt, J.W.; Copp, B.R.; Hu, W.P.; Munro, M.H.G.; Northcote, P.T.; Prinsep, M.R. Marine nature products. Nat. Prod. Rep. 2009, 26, 170-244.

3. Sims, J.J.; Lin, G.H.Y.; Wing, R.M. Marine natural products: Elatol, a halogenated sesquiterpene alcohol from the red alga Laurencia elata. Tetrahedron Lett. 1974, 39, 3487-3490. 
4. König, G.M.; Wright, A.D. Sesquiterpene content of the antibacterial dichlormethane extract of the red alga Laurencia obtusa. Planta Med. 1997, 63, 186-187.

5. Juagdan, E.G.; Kalidindi, R.; Scheuer, P. Two new chamigranes from an hawaiian red alga, Laurencia cartikzginea. Tetrahedron 1997, 2, 521-528.

6. Vairappan, C.S. Potent antibacterial activity of halogenated metabolites from Malaysian red algae, Laurencia majuscula (Rhodomelaceae, Ceramiales). Biomol. Eng. 2003, 20, 255-259.

7. Vairappan, C.S.; Anangdan, S.P.; Tan, K.L.; Matsunaga, S. Role of secondary metabolites as defense chemicals against ice-ice disease bacteria in biofouler at carrageenophyte farms. J. Appl. Phycol. 2009, 22, 305-311.

8. Lhullier, C.; Donnangelo, A.; Caro, M.; Palermo, J.A.; Horta, P.A.; Falkenberg, M.; Schenkel, E.P. Isolation of elatol from Laurencia microcladia and its palatability to the sea urchin Echinometra lucunter. Biochem. Syst. Ecol. 2009, 37, 254-259.

9. Granado, I.; Caballero, P. Chemical defense in the seaweed Laurencia obtusa (Hudson) Lamouroux. Sci. Mar. 1995, 59, 31-39.

10. De Nys, R.; Leya, T.; Maximilien, R.; Afsar, A.; Nair, P.S.R.; Steinberg, P.D. The need for standardised broad scale bioassay testing: A case study using the red alga Laurencia rigida. Biofouling 1996, 10, 213-224.

11. Vairappan, C.S.; Daitoh, M.; Suzuki, M.; Abe, T.; Masuda, M. Antibacterial halogenated metabolites from the Malaysian Laurencia species. Phytochemistry 2001, 58, 291-297.

12. König, G.M.; Wright, A.D. Laurencia rigida: Chemical investigations of its antifouling dichloromethane extract. J. Nat. Prod. 1997, 60, 967-970.

13. Santos, A.O.; Veiga-Santos, P.; Ueda-Nakamura, T.; Dias-Filho, B.T.; Sudatti, D.B.; Bianco, E.M.; Nakamura, C.V. Effect of Elatol, Isolated from Red Seaweed Laurencia dendroidea, on Leishmania amazonensis. Mar. Drugs 2010, 8, 2733-2743.

14. Veiga-Santos, P.; Pelizzaro-Rocha, K.J.; Santos, A.O.; Ueda-Nakamura, T.; Dias-Filho, B.T.; Silva, S.O.; Sudatti, D.B.; Bianco, E.M.; Nakamura, C.V. In vitro anti-trypanosomal activity of elatol isolated from red seaweed Laurencia dendroidea. Parasitology 2010, 137, 1661-1670.

15. Dias, T.; Brito, I.; Moujir, L.; Paiz, N.; Darias, J.; Cueto, M. Cytotoxic Sesquiterpenes from Aplysia dactylomela. J. Nat. Prod. 2005, 68, 1677-1679.

16. Campos, A.; Souza, C.B.; Lhullier, C.; Schenkel, E.P.; Ribeiro-do-Valle, R.M.; Siqueira, J.M. Antitumoral effects of elatol, a marine derivative compound obtained from red algae Laurencia microcladia. J. Pharm. Pharmacol. 2012, 64, 1146-1154.

17. González, A.G.; Darias, J.; Díaz, A.; Fourneron, J.D.; Martín, J.D.; Pérez, C. Evidence for the biogenesis of halogenated chamigrenes from the red alga Laurencia obtusa. Tetrahedron Lett. 1976, 17, 3051-3054.

18. Djuric, Z.; Heilbrun, L.K.; Lababidi, S.; Everett-Bauer, C.K.; Fariss, M.W. Growth inhibition of MCF-7 and MCF-10A human breast cells by $\alpha$-tocopheryl hemisuccinate, cholesteryl hemisuccinate and their ether analogs. Cancer Lett. 1997, 111, 133-139.

19. Kogure, K.; Manabe, S.; Hama, S.; Tokumura, A.; Fukuzawa, K. Potentiation of anti-cancer effect by intravenous administration of vesiculated $\alpha$-tocopheryl hemisuccinate on mouse melanoma in vivo. Cancer Lett. 2003, 192, 19-24. 
20. Bandyopadhyay, P.; Janout, V.; Zhang, L.; Regen, S.L. Ion conductors derived from cholic acid and spermine: Importance of facial hydrophilicity on $\mathrm{Na}^{+}$transport and membrane selectivity. J. Am. Chem. Soc. 2001, 123, 7691-7696.

21. Duran, F.J.; Ghini, A.A.; Dauban, P.; Dodd, R.H.; Burton, G. Synthesis of 6,19-sulfamidate bridged pregnanes. J. Org. Chem. 2005, 70, 8613-8616.

22. Mosmann, T. Rapid colorimetric assay for cellular growth and survival: Application to proliferation and cytotoxicity assays. J. Immunol. Methods 1983, 65, 55-63.

(C) 2012 by the authors; licensee MDPI, Basel, Switzerland. This article is an open access article distributed under the terms and conditions of the Creative Commons Attribution license (http://creativecommons.org/licenses/by/3.0/). 